\title{
Technical note: \\ Scintillations of the double star $\alpha$ Cru observed by GOMOS/Envisat
}

\author{
V. F. Sofieva ${ }^{1}$, F. Dalaudier ${ }^{2}$, V. Kan ${ }^{3}$, and A. S. Gurvich ${ }^{3}$ \\ ${ }^{1}$ Earth observation, Finnish Meteorological Institute, Helsinki, Finland \\ ${ }^{2}$ LATMOS, Université Versailles Saint-Quentin; CNRS/INSU, Verrières-le-Buisson, France \\ ${ }^{3}$ Organization of Russian Academy of Sciences A. M. Obukhov Institute of Atmospheric Physics RAS, Moscow, Russia
}

Received: 14 July 2009 - Published in Atmos. Chem. Phys. Discuss.: 2 September 2009

Revised: 12 November 2009 - Accepted: 14 November 2009 - Published: 27 November 2009

\begin{abstract}
In this paper, we discuss scintillation time-spectra of the double star $\alpha \mathrm{Cru}$, which were measured by the GOMOS/Envisat photometer. The components of $\alpha$ Cru are not resolved by the angular field of view of the detector. The double structure of the light source reveals itself in the modulation of the observed scintillation spectra; this modulation is caused by anisotropic irregularities of the stratospheric air density. We present a qualitative and quantitative explanation of the properties of the double-star scintillation spectra. Possibilities of using double star scintillations for studying atmospheric air density irregularities are also discussed in the paper.
\end{abstract}

\section{Introduction}

Statistical properties of scintillation (i.e. fluctuations in intensity when a light source is observed through the atmosphere) are defined by the structure of air density in the atmosphere, and by the spatial structure of the light source. Scintillations measured during star occultations have a broad application for carrying out research on the atmospheres of planets and their satellites (Hubbard et al., 1988; Raynaud et al., 2004), and they had been used actively during the last decade for satellite sounding of Earth air density irregularities (Gurvich and Kan, 2003a, b; Sofieva et al., 2007a, b). For the Earth's atmosphere, photometers with an effective aperture of $\sim 20 \mathrm{~cm}$ can register stellar scintillations in the altitude range $\sim 10-60 \mathrm{~km}$ with a vertical resolution down

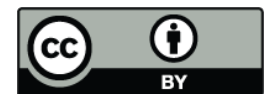

Correspondence to: V. F. Sofieva (viktoria.sofieva@fmi.fi) to meters/fraction of a meter. The theory of weak scintillation (Tatarskii, 1961, 1971) allows the analysis of scintillations measured aboard low-orbiting satellites for altitudes above $\sim 25-30 \mathrm{~km}$ (Gurvich and Kan, 2003a, b; Sofieva et al., 2007a, b).

Scintillations data with global coverage became available with the launch of the GOMOS (Global Ozone Monitoring by Occultation of Stars) instruments on board the Envisat satellite (Kyrölä et al., 2004). GOMOS is equipped with two fast photometers, which record the stellar flux synchronously in the blue $\left(473-527 \mathrm{~nm}, \lambda_{B} \approx 500 \mathrm{~nm}\right)$ and red $(646-698 \mathrm{~nm}$, $\lambda_{R} \approx 672 \mathrm{~nm}$ ) wavelength ranges with a sampling frequency of $1 \mathrm{kHz}$, as a star sets behind the Earth limb (for illustration of the occultation geometry, see Fig. 1 in Kyrölä et al., 2004). The scintillation measurements are used for the correction of scintillations in the spectrometer data to achieve a better accuracy of trace gases retrievals (Dalaudier et al., 2001; Sofieva et al., 2009b), for temperature profiling with a high vertical resolution (Dalaudier et al., 2006), and for studying small-scale processes in the stratosphere (Gurvich et al., 2005, 2007; Sofieva et al., 2007a, b; 2009a).

Recently, the algorithm for the reconstruction of gravity wave $(\mathrm{GW})$ and turbulence spectra parameters was developed (Sofieva et al., 2007b). During the data analyses, we have noticed that the scintillation spectra of $\alpha$ Cru are often radically different from those of other stars. This is illustrated in Fig. 1, which shows the scintillation spectra for the mean altitude of $35 \mathrm{~km}$. The left panel a shows the "standard" scintillation spectrum from occultations of Canopus. The majority of scintillation spectra of $\alpha$ Cru are quite different: a deep quasi-harmonic modulation of scintillation spectra is clearly observed (as in Fig. 1b). However, sometimes scintillation spectra of $\alpha$ Cru look normal (Fig. 1c). 

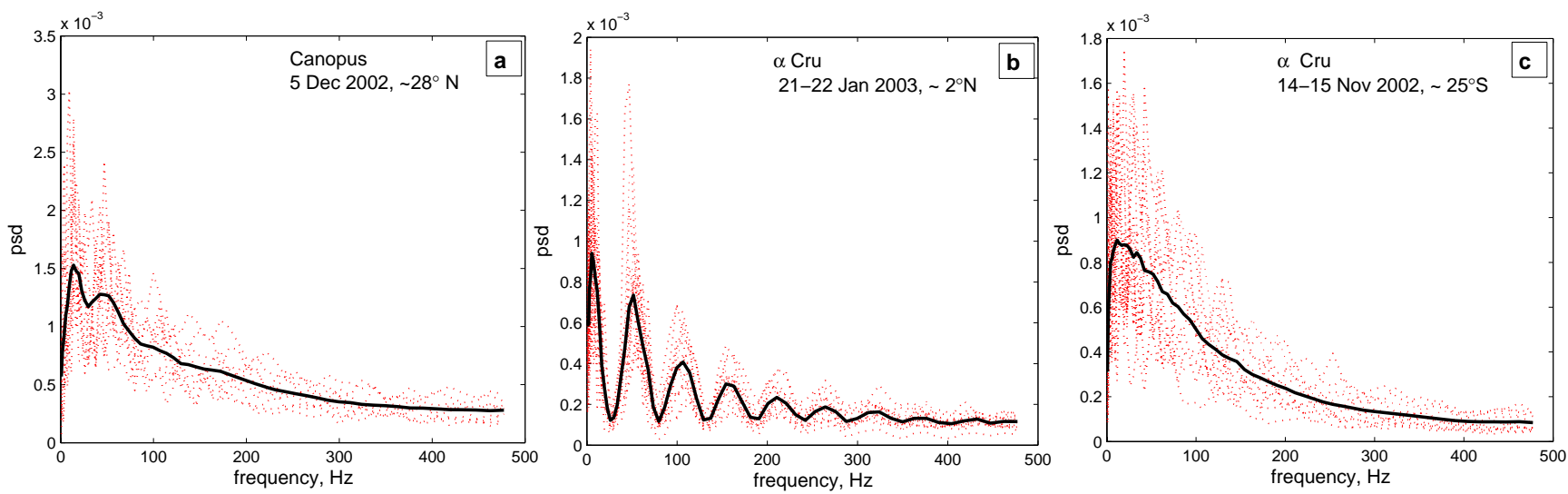

Fig. 1. (a): "standard" scintillation spectra (from occultations of Canopus); (b): scintillations spectra from several occultations of $\alpha$ Cru carried out on 21-22 January 2003; (c): scintillation spectra of $\alpha$ Cru on 14-15 November 2002 (usual spectra). Red lines: individual scintillation spectra estimations, black line: mean spectra, for altitude $35 \mathrm{~km}$. GOMOS successive occultations of each star are performed nearly at the same latitude and at nearly the same local time. Locations of the selected night-time occultations are indicated in the figure. Scintillation spectra are presented on a linear scale.

These features of the $\alpha$ Cru scintillation spectra are because $\alpha \mathrm{Cru}$ is a multiple (triple) star. Only two components of $\alpha \mathrm{Cru}$, which are separated by $\theta_{0}=4.4 \mathrm{arcsec}$, are visually distinguishable. They have comparable visual magnitudes $m v_{1}=1.4$ and $m v_{2}=2.09$. The GOMOS field of view is $138.4 \times 49.4$ arcsec (the larger angle is in the vertical direction), thus the components of $\alpha \mathrm{Cru}$ are not resolved by the detector. The intensity fields from the two components of $\alpha \mathrm{Cru}$, which are shifted with respect to each other, are registered simultaneously by GOMOS.

In this paper, we explain qualitatively and quantitatively the specific features and time evolution of scintillation spectra of double unresolved stars. The paper is organized as follows. Properties of scintillation spectra of double unresolved stars are explained in Sect. 2. Section 3 is dedicated to the comparison of experimental scintillation spectra with that computed using a two-component spectral model of air density irregularities. A discussion of the possible use of double star scintillations for studying atmospheric air density irregularities and a short summary conclude the paper.

\section{Scintillation spectra of double unresolved stars}

Satellite stellar scintillation measurements in the occultation geometry have been discussed in many papers (e.g. Gurvich, 1984; Dalaudier et al., 2001). We will use the phase screen approximation for the description of light wave propagation through the atmosphere (Little and Hewish, 1966). This means that the influence of the atmosphere is replaced by a plane screen that produces the same phase modulation of the propagated light waves. Such screen is placed in the plane, which is perpendicular to the incident light rays and contains the Earth's centre. For calculations of scintillation spectra of a double star with the components unresolved by a detector, let us take into account (Tatarskii, 1971, §42 and §54) that the components create distributions of intensity $I_{01} \mathrm{I}_{1}(\boldsymbol{r})$ and $I_{02} I_{2}(r)$ in the observation plane. Here $\boldsymbol{r}$ is the vector in this plane, $I_{01}$ and $I_{02}$ are spatially constant intensities without refractivity fluctuations, and $\mathrm{I}_{1}(\boldsymbol{r})$ and $\mathrm{I}_{2}(\boldsymbol{r})$ are random functions with mean value 1 . Provided the angular distance $\theta_{0}$ between the components is small $\theta_{0} \ll 1$, we can consider $\mathrm{I}_{2}(\boldsymbol{r})=\mathrm{I}_{1}(\boldsymbol{r}+\boldsymbol{\rho})$ (Little and Hewish, 1966; Cohen et al., 1967; Ishimaru, 1978), where the components of the vector $\boldsymbol{\rho}=\boldsymbol{\theta} D$ are $\rho_{z}=D \theta_{0, z} / q, \rho_{y}=D \theta_{0, y}, q$ is the refractive dilution (Dalaudier et al., 2001), $D$ is the distance between observation plane and the phase screen. In other words, the fields of relative intensity fluctuations corresponding to the components of a double star are only shifted with respect to each other. Then the 2-D spatial spectrum $F_{\Sigma}(\boldsymbol{\kappa})$ of the sum of the signals $\Sigma=I_{01} I_{1}(r)+I_{02} I_{2}(r)$ registered by the detector is equal to (the shifting theorem for the Fourier transform)

$F_{\Sigma}(\kappa)=\frac{I_{01}^{2}+I_{02}^{2}+2 I_{01} I_{02} \cos (\kappa \rho)}{\left(I_{01}+I_{02}\right)^{2}} F_{I}(\kappa)$,

where $F_{I}(\boldsymbol{\kappa}), \boldsymbol{\kappa}=\left(\kappa_{z}, \kappa_{y}\right)$ is the 2-D scintillation spectrum of a single star discussed in detail in (Gurvich and Brekhovskikh, 2001; Gurvich and Kan, 2003a; Sofieva et al., 2007b).

In occultation experiments, we obtain estimates of a 1-D scintillation spectrum along the occultation trajectory with an angle $\gamma$ to the local vertical (Gurvich and Brekhovskikh, 2001; Gurvich and Kan, 2003a):

$$
V_{\Sigma}\left(k_{s}, \gamma\right)=\int_{-\infty}^{+\infty} d k F_{\Sigma}\left(-k \sin \gamma+k_{s} \cos \gamma, k \cos \gamma+k_{s} \sin \gamma\right)
$$


The angle $\gamma=0$ corresponds to a vertical spectrum (and to a vertical, in-orbital-plane occultation). Therefore, the additional parameters that define properties of the scintillation spectra of double unresolved stars are: the ratio of intensities $I_{01} / I_{02}$ of the star components, the angular $\theta_{0}$ (or spatial $\rho$ ) separation, and the angle $\beta$ between $\rho$ and the local vertical.

As follows from (1), the scintillation variance for a double star is smaller than the variance of a single star of intensity $\left(I_{01}+I_{02}\right)$. Previously, the reduction of scintillation variance in the turbulent media caused by the finite angular size of the light source was already discussed by Tatarskii $(1961,1971)$. In particular, the known fact that planets scintillate less than stars for the same zenith angles has received its quantitative explanation in these works.

Another specific feature of unresolved double star scintillation spectra, which is caused by the factor $\cos (\kappa \rho)$ in the Eq. (1), is a modulation. The modulation of the 1-D scintillation spectra observed by GOMOS photometer depends not only on the orientation of the star components and occultation geometry (angles $\gamma$ and $\beta$ ), but also on the structure of atmospheric irregularities. It is known that there are two types of air density irregularities in the Earth atmosphere: anisotropic irregularities, which are of relatively large scale (generated by gravity waves), and isotropic irregularities (turbulence), which appear as a result of gravity wave breaking and due to different instabilities. The twocomponent spectral model of air density irregularities was proposed in (Gurvich and Brekhovskikh, 2001; Gurvich and Kan, 2003a). The scintillation spectra corresponding to this spectral model, for a single star, were discussed in detail in (Gurvich and Brekhovskikh, 2001; Gurvich and Kan, 2003a; Gurvich and Chunchuzov, 2005; Sofieva et al., 2007b). Due to the limited sampling frequency of GOMOS photometers, relatively large-scale anisotropic irregularities play an important role in forming the scintillations observed by GOMOS. As a result, scintillation spectra of $\alpha$ Cru are often modulated (Fig. 1b).

When oscillations in the 1-D anisotropic scintillation spectrum are observed, the modulation period $T_{m}$ in the frequency space increases with increasing $\beta$. It is defined by the vertical component of the projection of the satellite velocity onto the observation plane, $v_{v}$, and the vertical component of the stellar separation:

$T_{m}=v_{v} /(\rho \cos \beta)$.

The maximal modulation coefficient (contrast) for the anisotropic spectrum is

$m=\frac{2 I_{01} I_{02}}{I_{01}^{2}+I_{02}^{2}}$

For $\alpha$ Cru, which has two stars of similar intensities, $m$ is close to unity, $m \approx 0.9$.

\section{GOMOS observations of $\alpha$ Cru: a quantitative account}

In this section, we present the comparison of the experimental scintillation spectra of $\alpha \mathrm{Cru}$, from the GOMOS measurements, with that computed using the two-component spectral model of air density irregularities.

Information about four occultations of $\alpha \mathrm{Cru}$, selected for illustration, is collected in Table 1 . The angle $\beta$ changes from $\sim 90^{\circ}$ to $\sim 30^{\circ}$ during the selected time interval. The angle $\beta$ was computed using the information about vertical velocity and the observed modulation period of the scintillation spectra, Eq. (3). The experimental values of the angle $\beta$ agree well with the theoretical estimations of this parameter, which can be calculated using the occultation geometry. The spatial separation of $\alpha$ Cru components is $\rho=\theta_{0} D \approx 69 \mathrm{~m}$. The spatial sampling of GOMOS is from 3 to $8 \mathrm{~m}$, depending on obliquity of an occultation. For processing, we used scintillation measurements corresponding to an altitude of ray perigee of $\sim 34 \mathrm{~km}$, to satisfy the condition of weak scintillations (rms of scintillations are given in Table 1).

Figure 2 shows the experimental scintillation spectra for the selected occultations (red lines). The experimental spectra were computed using the method of smoothed periodograms. The periodogram smoothing was performed with the window of variable width, analogous to that discussed in (Gurvich and Chunchuzov, 2005; Sofieva et al., 2007b). Instrumental noise was removed in computing experimental scintillation spectra.

The width of anisotropic scintillation spectra is defined by the vertical inner scale $l_{W}: f_{a, \max }=v_{v} / l_{W}$ (see Table 1 ). For frequencies higher than $f_{a \text {, max }}$, the anisotropic scintillation spectra rapidly decrease. The strong modulation of the scintillation spectra at frequencies $f<f_{a, \max }$ for orbits 4677 , 4200 and 3945 (Fig. 2) is caused by prevailing anisotropic scintillations over isotropic ones in this spectral range. At frequencies $f>f_{a \text {, } \max }$, scintillations caused by isotropic irregularities prevail, thus the modulation of scintillation spectra rapidly decreases for $f>f_{a, \max }$.

Depending on $\beta$, the modulation period changes from $53 \mathrm{~Hz}$ for the orbit 4677 to $192 \mathrm{~Hz}$ for the orbit 3945 . For the orbit 3705, the components of $\alpha$ Cru are located nearly horizontally, their vertical separation was much smaller than $l_{W}$. In this case, the anisotropic component of the scintillation spectrum is nearly the same as for a single star, while the spectral density of the isotropic component is approximately twice as small as that of a single star. The observed scintillation spectrum in this case is not modulated, as seen in Fig. 2.

Black lines in Fig. 2 show the fit of the experimental spectral to the modeled scintillation spectra, which were computed using the two-component spectral model of air density irregularities, including anisotropic (saturated gravity waves) and isotropic (Kolmogorov turbulence) components (Gurvich and Kan, 2003a; Sofieva et al., 2007b). A brief 

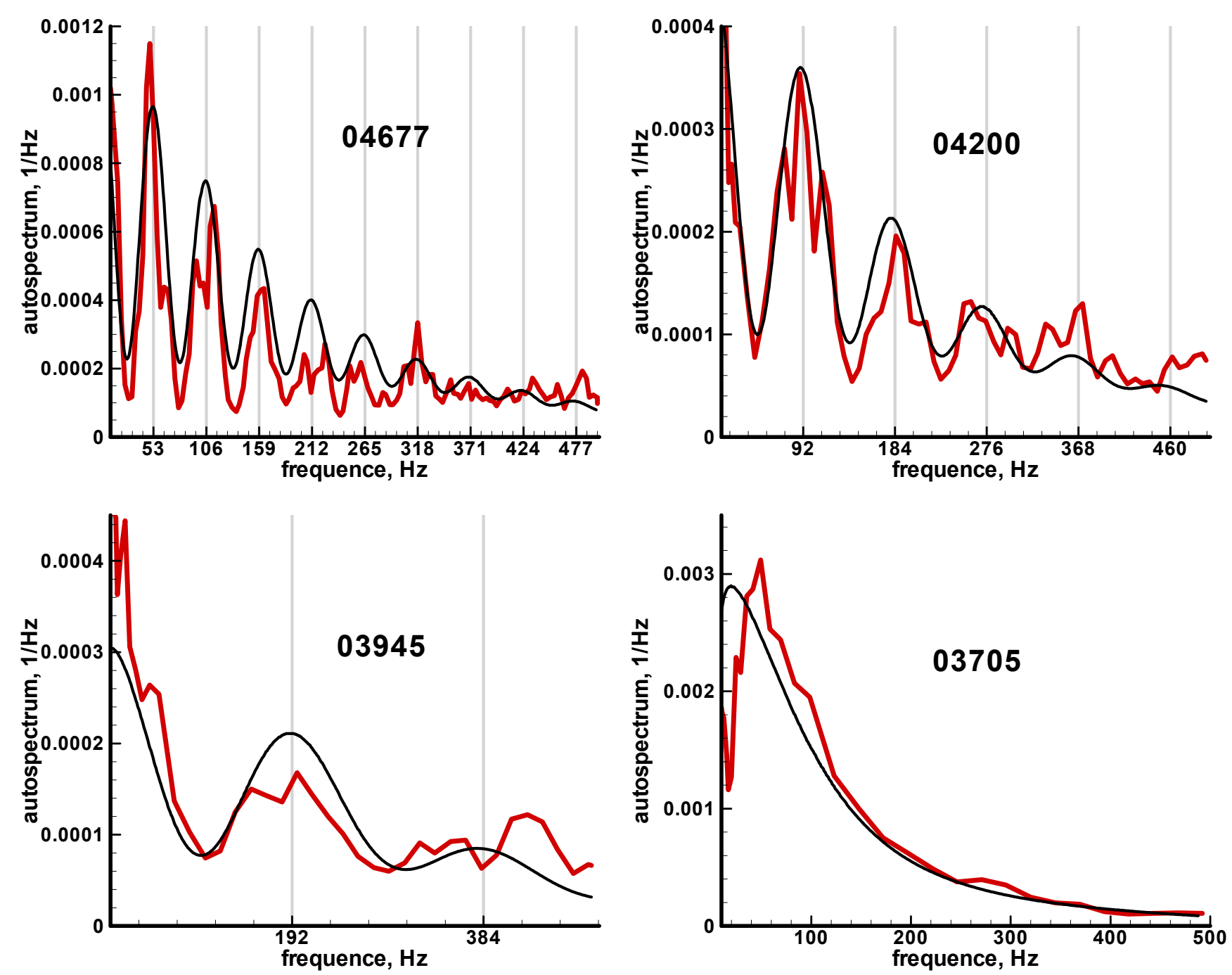

Fig. 2. Experimental scintillation spectra on a linear scale of $\alpha$ Cru (red lines) and their fit using the two-component spectral model of air density irregularities (black lines). Vertical gray lines correspond to modulation periods $T_{m}$.

description of this model is given in Appendix A. The computation of theoretical scintillation spectra was discussed in detail in (Gurvich and Brekhovskikh, 2001; Gurvich and Chunchuzov, 2005; Sofieva et al., 2007b). This model and Eqs. (1) and (2) were used for fitting 1-D modeled spectra to the observations. The estimates of the three parameters of the spectral model - the structure characteristic $C_{W}$ of anisotropic irregularities, vertical inner scale $l_{W}$, and the structure characteristic $C_{K}$ of Kolmogorov turbulence - retrieved in the fitting, are presented in Table 1. As seen in Fig. 2, the modeled and experimental scintillation spectra are in rather good agreement. The obtained estimates of gravity waves and turbulence parameters are within the range of previous results of the parameter reconstruction (Gurvich and Kan, 2003b; Sofieva et al., 2007b, 2009a).

\section{Summary and discussion}

We have considered the properties of scintillation spectra of double stars, which are not resolved by the field of view of the detector. The main features of the double-star scintillation are (i) reduction of scintillation variance (compared to that of a single star) and (ii) modulation of the scintillation spectra. It is the consequence of shifted intensity fields corresponding to the components of a double star. The period and the depth of the modulation depend on the structure of atmospheric irregularities, separation of the star components, ratio of their intensities, and on the observation geometry. The modulation of the observed scintillation spectra is caused mainly by anisotropic irregularities of air density (due to limited resolution of the GOMOS fast photometers, such irregularities play an important role in forming the scintillations observed by GOMOS). 
Table 1. GOMOS occultations selected for the comparison.

\begin{tabular}{|c|c|c|c|c|c|c|c|c|c|}
\hline $\begin{array}{c}\text { Date and } \\
\text { orbit }\end{array}$ & $\begin{array}{c}\text { Ray } \\
\text { perigee } \\
\text { coordinates }\end{array}$ & $\begin{array}{l}\text { Scintillation } \\
\text { rms, } \%\end{array}$ & $\begin{array}{c}\gamma \\
\text { deg }\end{array}$ & $\begin{array}{c}\beta \\
\operatorname{deg}\end{array}$ & $\begin{array}{l}\text { Modulation } \\
\text { period } \mathrm{T}_{m} \\
(\mathrm{~Hz})\end{array}$ & $\begin{array}{c}f_{a, \max } \\
(\mathrm{Hz})\end{array}$ & $\begin{array}{c}C_{W} \cdot 10^{11}, \\
\mathrm{~m}^{-2}\end{array}$ & $\begin{array}{c}l_{W} \\
\mathrm{~m}\end{array}$ & $\begin{array}{c}C_{K} \cdot 10^{9}, \\
\mathrm{~m}^{-2 / 3}\end{array}$ \\
\hline $\begin{array}{c}21 \text { Jan } 2003 \\
\quad \# 04677\end{array}$ & $\begin{array}{l}1.5^{\circ} \mathrm{N} \\
45.3^{\circ} \mathrm{E}\end{array}$ & 35 & 34.5 & 31.2 & 53 & 210 & 8.25 & 15 & 5.8 \\
\hline $\begin{array}{c}19 \text { Dec } 2002 \\
\# 04200\end{array}$ & $\begin{array}{c}14.3^{\circ} \mathrm{S} \\
162.6^{\circ} \mathrm{E}\end{array}$ & 25 & 23.0 & 59.3 & 92 & 215 & 3.7 & 15 & 2.2 \\
\hline $\begin{array}{c}1 \text { Dec } 2002 \\
\# 03945\end{array}$ & $\begin{array}{l}21.2^{\circ} \mathrm{S} \\
94.4^{\circ} \mathrm{E}\end{array}$ & 26 & 9.4 & 75.5 & 192 & 470 & 2.5 & 7 & 1.4 \\
\hline $\begin{array}{c}14 \text { Nov } 2002 \\
\text { \# } 03705\end{array}$ & $\begin{array}{l}25.5^{\circ} \mathrm{S} \\
7.7^{\circ} \mathrm{E}\end{array}$ & 64 & -7.1 & $\sim 90$ & $>500$ & 130 & 28.9 & 25 & 4.3 \\
\hline
\end{tabular}

The experimental scintillation spectra obtained by GOMOS in occultations of the double star $\alpha$ Cru under different observation conditions are adequately (qualitatively and quantitatively) explained, if existence of anisotropic and isotropic irregularities of air density is assumed. Therefore, the performed analysis can serve as an additional confirmation of the existence of such irregularities in the atmosphere.

The scintillation spectra of double stars are more complicated compared to that of a single star, but they are more informative. For example, in (Gurvich and Kan, 2003a, b; Sofieva et al., 2007b), the amplitude of the isotropic component (and the turbulent structure characteristic $C_{K}$ ) was estimated using the high-frequency part of the scintillation spectra $f>f_{a, \max }$. Although the reconstruction of gravity wave and turbulence spectra parameters is formulated via the solution of the corresponding inverse problem in (Sofieva et al., 2007b), the information about the turbulent component is actually contained only in the high-frequency part of the scintillation spectrum, $f>f_{a, \max }$. For occultations close to vertical and /or provided the inner scale $l_{W}$ of the anisotropic component is small, the turbulence parameters cannot be retrieved reliably. For double stars, the detection of the isotropic component (and thus its parameters) can be done in the whole spectral range.

The availability of two photometers at different wavelengths allows a simultaneous sounding of the atmosphere along the trajectories separated in the vertical direction due to chromatic refraction. The chromatic shift between the sounding trajectories is defined by the mean refractivity; it depends on altitude. For GOMOS photometers, it does not exceed $10 \mathrm{~m}$ above $30 \mathrm{~km}$ (Dalaudier et al., 2001). Gurvich et al. (2005) have shown that bi-chromatic scintillations open new possibilities in studies of air density irregularities by using the chromatic effects. In particular, it was shown that the coherency spectra and cross-correlation function can be used for indicating regions with high intensity of isotropic turbulence. For a double star, the two GOMOS photometers perform the sounding of the atmosphere along four separated trajectories. The chromatic separation is variable (in the vertical direction), while the separation of stars can be in any direction. Smooth changes of occultation obliquity $\gamma$ and the angle of stellar separation with respect to the local vertical $\beta$ in successive occultations (due to satellite precession), can provide data with the distance between the sounding trajectories ranging from zero to $\rho$ (depending on $\gamma$ and $\beta$ ). A feasibility study on using double star scintillations for obtaining information about the structure of air density irregularities might be carried out in the future.

\section{Appendix A}

\section{The spectral model of refractivity irregularities in the atmosphere. Scintillation spectra}

For the optical wavelength range, relative fluctuations of refractivity $\nu=\delta N /\langle N\rangle$ ( $N$ is refractivity, angular brackets denote the statistical mean) are equal to relative fluctuations of air density. $\langle N\rangle$ depends on altitude with the atmospheric scale height of $6-8 \mathrm{~km}$. The dependence $\langle N\rangle$ on wavelength is defined by the known dispersion relation for air.

The properties of the refractivity field are characterized by the 3-D spectrum $\Phi_{v}(\kappa)$ of relative refractivity fluctuations, $\boldsymbol{\kappa}=\left(\kappa_{x}, \kappa_{y}, \kappa_{z}\right)$ being a wave vector $(z$ is the vertical coordinate). Following (Gurvich and Brekhovskikh, 2001; Gurvich and Kan, 2003a), we assume that $\Phi_{\nu}$ is the sum of two statistically independent components:

$\Phi_{\nu}=\Phi_{W}+\Phi_{K}$

The anisotropic component $\Phi_{W}$ corresponds to the saturated gravity wave model (Smith et al., 1987). It has the slope -5 between the inner scale $l_{W}$ and the outer scale $L_{W}$. The structure characteristic $C_{W}$ defines the power of anisotropic irregularities. The anisotropy coefficient $\eta$ is defined as a ratio of 
the characteristic horizontal and vertical scales. It is assumed that $\eta=$ const $\gg 1$ (Gurvich and Brekhovskikh, 2001; Gurvich and Kan, 2003a; Sofieva et al., 2007b). The associated onedimensional vertical spectrum has the slope -3 , as in the model of saturated gravity waves. The isotropic component $\Phi_{K}$ corresponds to locally isotropic turbulence (Monin and Yaglom, 1975) with the slope $-11 / 3$ of power spectral density. It has two parameters: the structure characteristic $C_{K}$ and the inner (Kolmogorov) scale $l_{K}$. The one-dimensional spectrum corresponding to $\Phi_{K}$ follows the well-known $-5 / 3$ power law.

The scintillation spectra at the observation plane can be computed using the theory of wave propagation in a random media. The main approximations used in the evaluation of the scintillation spectra are:

- frozen field approximation;

- phase screen approximation;

- weak scintillation assumption (i.e. rms of scintillation is significantly smaller than 1 ).

The computation of theoretical scintillation spectra is discussed in detail in (Gurvich and Brekhovskikh, 2001; Gurvich and Kan, 2003a; Sofieva et al., 2007b).

Since the inner vertical scale $l_{W}$ varies from $\sim 10 \mathrm{~m}$ to a few tens of meters in the stratosphere, $l_{W}>\rho_{F}$ for satellite measurements, where $\rho_{F}=\sqrt{\lambda D / 2 \pi}$ is the Fresnel scale, ( $\rho_{F} \approx 0.5 \mathrm{~m}$ for GOMOS). In this case, the diffractive effects are not important and the vertical scale of scintillations, which are generated by anisotropic irregularities, is defined by $l_{W}$. In contrast, scintillations caused by isotropic irregularities are defined by the diffractive effects when $\rho_{F}>l_{K}$. In this case, the characteristic scale of isotropic scintillations is the Fresnel scale. Scintillations caused by Kolmogorov turbulence are not sensitive to the outer scale $L_{K}$ of turbulence (Tatarskii, 1961, 1971; Gurvich and Chunchuzov, 2005).

Acknowledgements. The authors thank V. V. Vorobjev for useful discussions and comments. The authors thank ESA, ACRI-ST and the GOMOS team for the GOMOS data. The work of V. Kan and A. S. Gurvich was supported by Russian Foundation for Basic Research, grant 09-05-00180. The work of V. F. Sofieva was supported by the Academy of Finland.

Edited by: P. Bernath

\section{References}

Cohen, M. H., Gundermann, E. J., and Harris, D. E.: New limits on the diameters of radio sources, Astrophys. J., 150, 767-782, 1967.

Dalaudier, F., Kan, V., and Gurvich, A. S.: Chromatic refraction with global ozone monitoring by occultation of stars, I. Description and scintillation correction, Appl. Optics, 40, 866-877, 2001.
Dalaudier, F., Sofieva, V., Hauchecorne, A., Kyrölä, E., Blanot, L., Guirlet, M., Retscher, C., and Zehner, C.: High-resolution density and temperature profiling in the stratosphere using bichromatic scintillation measurements by GOMOS, Proceedings of the First Atmospheric Science Conference, European Space Agency, ISBN 92-9092-939-1-ISSN 1609-042X, 2006.

Gurvich, A. S.: Fluctuation during observation of extraterrestrial sources from space through atmosphere of the Earth, Radiophys. Quantum El., 27, 665-673, 1984.

Gurvich, A. S. and Brekhovskikh, V. L.: Study of the turbulence and inner waves in the stratosphere based on the observations of stellar scintillations from space: a model of scintillation spectra, Waves Random Media, 11, 163-181, 2001.

Gurvich, A. and Chunchuzov, I.: Estimates of characteristic scales in the spectrum of internal waves in the stratosphere obtained from space observations of stellar scintillations, J. Geophys. Res., 110, D03114, doi:10.1029/2004JD005199, 2005.

Gurvich, A. S., Dalaudier, F., and Sofieva, V. F.: Study of stratospheric air density irregularities based on two wavelength observation of stellar scintillation by Global Ozone Monitoring by Occultation of Stars (GOMOS) on Envisat, J. Geophys. Res., 110, D11110, doi:10.1029/2004JD005536, 2005.

Gurvich, A. S. and Kan, V.: Structure of Air Density Irregularities in the Stratosphere from Spacecraft Observations of Stellar Scintillation, 1. Three-Dimensional Spectrum Model and Recovery of Its Parameters, Izvestiya, Atmos. Ocean. Phys., 39, 300-310, 2003a.

Gurvich, A. S. and Kan, V.: Structure of air density irregularities in the stratosphere from spacecraft observations of stellar scintillation: 2. Characteristic scales, structure characteristics, and kinetic energy dissipation, Izvestiya, Atmos. Ocean. Phys., 39, 311-321, 2003b.

Gurvich, A. S., Sofieva, V. F., and Dalaudier, F.: Global distribution of $\mathrm{C}_{T}^{2}$ at altitudes $30-50 \mathrm{~km}$ from space-borne observations of stellar scintillation, Geophys. Res. Lett., 34, L24813, doi:10.1029/2007GL031134, 2007.

Hubbard, W. B., Lellouch, E., Sicardy, B., Brahic, A., Vilas, F., Bouchet, P., McLaren, R. A., and Perrier, C.: Structure of scintillations in Neptune's occultation shadow, Astrophys. J., 325, 490-502, 1988.

Ishimaru, A.: Wave propagation and scattering in random media, V. 2. Multiple scattering, turbulence, rough surfaces and remote sensing, Academic Press, 572 pp., 1978.

Kyrölä, E., Tamminen, J., Leppelmeier, G. W., et al.: GOMOS on Envisat: An overview, Adv. Space Res., 33, 1020-1028, doi:10.1016/S0273-1177(03)00590-8, 2004.

Little, L. T. and Hewish, A.: Interplanetary scintillation and its relation to the angular structure of radio sources, Mon. Not. R. Astron. Soc., 134, 221-237, 1966.

Monin, A. S. and Yaglom, A. M.: Statistical fluid mechanics, v.2, MIT Press, Cambridge, Mass., 1975.

Raynaud, E., Matheva, K., Drossart, P., Roques, F., and Sicardy, B.: A reanalysis of the 1971 Beta Scorpii occultation by Jupiter: study of the temperature fluctuations and detection of wave activity, Icarus, 168, 324-335, doi:10.1016/j.icarus.2003.10.021, 2004.

Smith, S. A., Fritts, D. C., and VanZandt, T. E.: Evidence of a saturation spectrum of atmospheric gravity waves, J. Atmos. Sci., 44, 1404-1410, 1987. 
Sofieva, V. F., Kyrölä, E., Hassinen, S., et al.: Global analysis of scintillation variance: Indication of gravity wave breaking in the polar winter upper stratosphere, Geophys. Res. Lett., 34, L03812, doi:10.1029/2006GL028132, 2007a.

Sofieva, V. F., Gurvich, A. S., Dalaudier, F., and Kan, V.: Reconstruction of internal gravity wave and turbulence parameters in the stratosphere using GOMOS scintillation measurements, J. Geophys. Res., 112, D12113, doi:10.1029/2006JD007483, 2007b.

Sofieva, V. F., Gurvich, A. S., and Dalaudier, F.: Gravity wave spectra parameters in 2003 retrieved from stellar scintillation measurements by GOMOS, Geophys. Res. Lett., 36, L05811, doi:10.1029/2008GL036726, 2009a.
Sofieva, V. F., Kan, V., Dalaudier, F., Kyrölä, E., Tamminen, J., Bertaux, J.-L., Hauchecorne, A., Fussen, D., and Vanhellemont, F.: Influence of scintillation on GOMOS ozone retrievals, Atmos. Chem. Phys. Discuss., 9, 12615-12643, 2009b .

Tatarskii, V. I.: Wave propagation in a turbulent medium, McGrawHill, New York, 285 pp., 1961.

Tatarskii, V. I.: The effects of turbulent atmosphere on wave propagation, National Technical Information Service, Springfield, Va., 417 pp., 1971. 